\title{
Flow Augmenting Routing with Network Coding for Lifetime Maximization in Wireless Networks
}

\author{
Lianghui Ding ${ }^{1}$, Ping $\mathrm{Wu}^{1}$, Hao Wang ${ }^{2,1}$, Zhiwen $\mathrm{Pan}^{2}$, Xiaohu You ${ }^{2}$ \\ ${ }^{1}$ Signals and Systems, Dept. of Engineering Sciences, Uppsala University, Uppsala, Sweden \\ ${ }^{2}$ National Mobile Communication Research Laboratory, Southeast University, Nanjing, China \\ \{lhding, ping.wu\}@angstrom.uu.se, \{hao_wang, pzw, xhyu\}@seu.edu.cn
}

\begin{abstract}
The lifetime maximization routing with network coding in wireless multihop networks is considered in this paper. The problems is formulated in three different cases: (i) no network coding, (ii) two-way network coding, and (iii) overhearing network coding. Flow augmenting routing (FA) is used to solve the first case, and then it is extended to FA with network coding (FANC) for the latter two. The performance of FANC with two-way and overhearing network coding schemes is evaluated and compared with that without network coding under two different power control models, namely, protocol and physical ones. The results show that the lifetime can be improved up to $23 \%$ and $8 \%$ in a random network under the protocol and physical models, respectively. It is also found that the lifetime improvement ratio increases as the number of traffic demands under the protocol model, while it increases first, and then decreases under the physical model.
\end{abstract}

\section{INTRODUCTION}

In energy constrained wireless multihop networks, such as wireless sensor networks, replacing or recharging batteries of nodes is often inconvenient or even impossible, and their lifetime is limited by the available energy of network nodes. Thus, lifetime maximization has been an interesting research topic and investigated extensively in recent years. Up to now a variety of methods have been proposed to prolong the network lifetime [1], [2].

The concept of network coding was first constructed by Ahlswede in [3], wherein it was proved that the achievable rate region of multicast is the max-flow/max-cut from the source to sink nodes, and can be realized with block coding. After that, a lot of research has been done on various aspects of network coding, such as coding approaches [4], [5], practical application of network coding [6]-[8], network coding for energy efficiency [9], etc.

It has been known that network coding can effectively improve the throughput [3], [6] and total energy efficiency [9] of both wired and wireless networks. The basic idea of network coding is to equip routers with processing ability, and compress information utilizing spatial redundancy among nodes to reduce the occupied network bandwidth. Since wireless signal is inherently broadcast, and there is sufficient spatial redundancy that can be used by network coding, much research

This work is supported mainly by VINNOVA Sweden (Grant 2008- 00954); and in part by CSC China, the Ministry of Science and Technology China through International Science and Technology Cooperation Program under grant 2008DFA12090 and National Communications Research Laboratory Program (2009A02). has been done to improve the network performance with network coding in wireless networks [6].

However, few research has been done on lifetime maximization with network coding in wireless multihop networks. In intuition, employment of network coding can decrease the number of transmissions, and thus save the power of some bottleneck nodes and prolong the lifetime of the whole network. Some research has been done in this area. Lifetime extension with network coding in some specific scenarios is presented in [10]. Problem formulation of lifetime maximized multicast with network coding is given in [11], [11]. However, no reports have been found on lifetime maximization with inter-session network coding, while it has been known that routing selection heavily affects the network lifetime [1], and the network throughput with network coding [7].

In this paper, therefore, we investigate the lifetime maximization routing with inter-session network coding in wireless multihop networks. Three problems for lifetime maximization are formulated with no network coding, two-way network coding, and overhearing network coding. Then flow augmenting routing with network coding (FANC) is proposed to solve the latter two problems. The performance of FANC is evaluated under both protocol and physical power control models and compared with that of Flow Augmenting (FA) routing with no network coding.

The rest of the paper is organized as follows. We first give definitions and notations of network model, inter-session network coding, and the network lifetime in Section II. Then we present problem formulation of lifetime maximization for three different cases in Section III. The flow augmenting routing with network coding (FANC) is is proposed and evaluated in Sections IV and V, respectively. Finally the paper is concluded in Section VI.

\section{Preliminaries}

\section{A. Network Model}

A wireless multihop network is modeled as a directed graph $G=\{V, E\}$, where $V$ is the set of nodes, $E$ is the set of links. Links are assumed to be symmetric here, i.e. if link $(i, j) \in E$, then there exits $(j, i) \in E$. The commodity is defined as a unicast traffic from a source node to a sink node. Throughout the whole paper, we use $c$ and $C$ to denote a specific commodity and the set of all commodities, respectively, and use $s^{c}$ and $d^{c}$ to denote the source and destination nodes of commodity $c$. 
Transmission power control is an effective technology to reduce power cost and spatial interference, and perfect power control is often assumed in previous analysis of lifetime [1]. However, only limited power control is supported in a practical system. Thus, in this paper, we consider two extreme states. One is without any transmission power adjustment, named as protocol model, while another is with perfect power control, named as physical model.

Let $\eta$ denote the threshold of the received signal to interference and noise ratio (SINR) to correctly decode a packet. Then in the protocol model, the node always transmits with the maximum power, and transmission of each bit costs the same power. In the physical model, node $j$ can correctly receive the data from node $i$ if the received SINR is larger than the threshold $\eta$, and the power cost of one bit transmission depends on the distance between the transmitter and receiver.

Under a certain interference model for resource sharing and scheduling, e.g, protocol or physical models, the achievable rate region of the network $G$ can be defined as $C o(r)$, which is a multi-dimension polyhedron.

\section{B. Network Coding}

Although there have been plenty of network coding approaches in literature [4], [5], [12], one-hop linear network coding, COPE [6], is a practical sub-optimal inter-session network coding with significant performance improvement, low overhead and easy extension. However, the percentage of network coding with 2 symbols is more than 50\% in COPE [6], and more than $1 \%$ in COPR [9]. Furthermore, from the practical observation, considering network coding with more than 2 symbols will seriously complicate the optimization process. Thus, in this paper, we will focus our analysis on the one-hop linear network coding with only 2 symbol using linear XOR, which is similar to $c=a \oplus b$. Here, $a$ and $b$ are input packets, and $c$ is the output packet after XOR.

The requirement of one-hop network coding is given in the following. In one-hop network coding, the coded packet $P_{c}=$ $P_{1} \oplus P_{2}$ including 2 uncoded packets transmitted by node $i$ can be decoded by nodes $j$ if and only if: (1) Node $j$ correctly receives the packet $P_{c}$ from $i$; (2) Node $j$ has obtained one packet coded in $P_{c}$.

We consider two opportunities that node $j$ can obtain packets for decoding $P_{c}$ from $i$ : (1) $j$ is the last hop of the packet. For instance, the packet $P_{x}$ at node $x$ and $P_{z}$ at node $z$ in Figure 1(a); (2) Node $j$ overhears the packet from link $(k, i)$, $k \neq j$, such as the packet $P_{t}$ received by node $v$ in Figure 1(b). According to packets allowed in one-hop inter-session network coding, we give definitions of two-way network coding and overhearing network coding as follows.

Definition 1 (Two-Way Network Coding) One-hop network coding only allowing coding packets from the opportunity (1).

Definition 2 (Overhearing Network Coding) One-hop network coding considering coding opportunities (1) and (2).
Besides the two opportunities presented above, there are three others that $j$ can obtain packet $P_{k}$ : (i) node $j$ gets $P_{k}$ through link $(j, k), k \neq i$; (ii) $j$ overhears $P_{k}$ from link $(k, l)$, $l \neq i$; (iii) $j$ overhears coded packets include $P_{k}$ and decodes it, such as node $w$ overhears $P_{s} \oplus P_{t}$ and decodes it with the earlier overheard packet $P_{t}$ in Figure 1(b). Since these three coding scenarios happen rarely and require more buffer space at node $j$ [7], we do not consider them here.

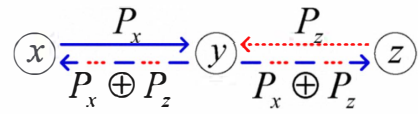

(a) Two-way network coding.

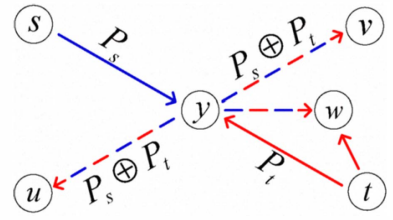

(b) Overhearing and decoding
Fig. 1. Illustration of one-hop network coding.

Since the one-hop network coding depends on the previous hop where the data comes from, we define a two-hop link in this paper for expression facilitation.

Definition 3 (Two-Hop Link) For node i, links from the node $j$ to the node $k$ through node $i, j \neq k$, is defined as the two-hop link at node $i$ and written as $(j, i, k)$.

Note that for the source node, $j=i$, while for the destination, there exists $i=k$. The one-hop network coding at node $i$ depends on the local flow distribution through it and relative locations of its neighboring nodes. As shown in Figure 1(b), the two-hop links $(s, y, v),(t, y, u)$ can be coded, while $(s, y, u)$, $(t, y, v)$ can not be coded together. We use $S_{i}$ to denote a possible network coding scheme at node $i$, and use $F_{i}^{2}, F_{i}^{o}$, $F_{i}=\left\{F_{i}^{2}, F_{i}^{o}\right\}$ to denote the union of scenarios of two-way network coding, overhearing network coding, and all network coding scenarios, respectively.. For convenience, we consider the transmission of uncoded packets without network coding as a special network coding scheme with only one flow. We use $p\left(S_{i}\right)$ and $n\left(S_{i}\right)$ to denote the previous and next hop node sets of $S_{i}$, respectively. For instance, the previous and next hop node sets of $S_{i}=\left\{\left(j_{1}, i, k_{1}\right),\left(j_{2}, i, k_{2}\right)\right\}$ are $p\left(S_{i}\right)=\left\{j_{1}, j_{2}\right\}$, $n\left(S_{i}\right)=\left\{k_{1}, k_{2}\right\}$.

\section{Network Lifetime}

The network lifetime is the time period during which the network functions properly, in particular, all demands can be satisfied. To make the analysis more tractable, we use the shortest lifetime of all nodes as that of the network. In the following the formal definition is given.

Let $E_{i}$ denote the total energy of node $i$, and $e_{S_{i}}^{t}$ denote the power cost of transmitting a data unit in $S_{i}$. Let $e_{j i}^{r}$ and $e_{i k}^{t}$ denote the receiving power cost of node $i$ from $j$, and the transmission power cost from node $i$ to $k$, respectively. For a uncoded transmission with only one two-hop link $S_{i}=(j, i, k)$, $e_{S_{i}}^{t}=e_{i k}^{t}$. For a network coding scheme $S_{i}$ with more twohop links, the transmission power cost is $e_{S_{i}}^{t}=\max _{k \in n\left(S_{i}\right)}\left\{e_{i k}^{t}\right\}$. 
For any network coding scheme with $i \in n\left(S_{j}\right)$, the receiving power cost is $e_{j i}^{r}$.

Let $q_{S_{i}}^{c}$ denote the physical transmission rate of commodity $c$ through $S_{i}$, then the power cost in a unit time at node $i$ for transfer of commodity $c$ is

$P_{i}^{c}=\sum_{S_{i} \in F_{i}} q_{S_{i}}^{c} e_{S_{i}}^{t} /\left|S_{i}\right|+\sum_{j \in V} \sum_{\substack{S_{j} \in F_{j} \\ i \in n\left(S_{j}\right)}} q_{S_{j}}^{c} e_{j i}^{r}+\sum_{j \in V} \sum_{\substack{S_{j} \in F_{j}^{o} \\ i \in n\left(S_{j}\right), i \nsubseteq \neq p\left(S_{j}\right)}} q_{S_{j}}^{c} e_{j i}^{r}$

where the first term is the transmission power cost at node $i$, the second is the receiving power cost of transmission of commodity $c$ from all neighboring nodes $j$, and the third term is the receiving power cost for overhearing. Note that the condition of overhearing at node $i$ is $i \in n\left(S_{j}\right)$, while $i \not p\left(S_{j}\right)$.

Then the lifetime of node $i$ can be expressed as

$$
T_{i}=\frac{E_{i}}{\sum_{c \in C} P_{i}^{c}}
$$

And the lifetime of the network is given by

$$
T=\min _{i \in V} T_{i}
$$

Note that lifetime maximization routing will distribute flow demands to all nodes, and the lifetime obtained from $\max T$ is equivalent to the definition that the network is not connected anymore after reaching the lifetime limit.

\section{Problem Formulation}

\section{A. No Network Coding}

Without network coding, the lifetime maximization can be formulated as follows.

$$
\begin{gathered}
\max T \\
\text { s.t. } \sum_{(i, j) \in E} q_{i j}^{c}-\sum_{(j, i) \in E} q_{j i}^{c}=\sigma_{i}^{c}, \quad \forall i, c \\
\left(\sum_{c \in C} \sum_{(i, j) \in E} e_{i}^{t} q_{i j}^{c}+\sum_{c \in C} \sum_{(j, i) \in E} e_{i}^{r} q_{j i}^{c}\right) T \leq E_{i}, \quad \forall i \\
\sum_{c \in C} q_{i j}^{c} \leq r_{i j}, \quad \forall\{i, j\} \\
\left(r_{i j}\right) \in C o(r)
\end{gathered}
$$

where equation (4) is the flow conservation constraint: at each node, the total output rates of commodity $c$ are equal to the corresponding input rates. Here, $q_{i j}^{c}$ denotes the transmission rate from node $i$ to node $j$ of commodity $c$. The rate $\sigma_{i}^{c}$ is

$$
\sigma_{i}^{c s}=\left\{\begin{array}{cl}
x^{c}, & \text { if } i=s^{c} \\
-x^{c}, & \text { if } i=d^{c} \\
0, & \text { otherwise. }
\end{array}\right.
$$

where $x^{c}$ is the traffic demand of the commodity $c$.

Equation (5) is the energy constraint, which tells the energy cost of node $i$ in lifetime $T$ should be less than its total energy $E_{i}$. Rate constraints are given in equations (6)(7). Total rate passed through link $(i, j)$ should be less than scheduled rate $r_{i j}$, and all link rates are in the achievable rate region $C o(r)$, which depends on link rates and the interference model, etc.

When link rates are large enough that all demands can be supported by all possible paths simultaneously, interference and scheduling will not influence the performance of lifetime. Since it is often true in an energy constrained wireless network with relatively small traffic demands, we can omit the corresponding rate constraints in equations (6)(7), and constraints are simplified to the formulation in [13].

$$
\begin{gathered}
\sum_{(i, j) \in E} \hat{g}_{i j}^{c}-\sum_{(j, i) \in E} \hat{g}_{j i}^{c}=T \sigma_{i}^{c}, \quad \forall i, c \\
\left(\sum_{c \in C} \sum_{(i, j) \in E} e_{i}^{t} \hat{g}_{i j}^{c}+\sum_{c \in C} \sum_{(j, i) \in E} e_{i}^{r} \hat{g}_{j i}^{c}\right) \leq E_{i}, \quad \forall i
\end{gathered}
$$

where $\hat{g}_{i j}^{c}=T q_{i j}^{c}$ is the total amount of data of commodity $c$ transmitted on link $(i, j)$ in lifetime $T$. When $T$ is considered as an independent variable, the lifetime maximization problem becomes to a linear programming problem [13].

In the following, we assume link rates are large enough, and only consider the influence of total flow distribution on lifetime maximization routing.

\section{B. Two-Way Network Coding}

For two-way network coding, only network coding scheme $S_{i}=\{(j, i, k),(k, i, j)\}, j \neq i \neq k$ and uncoded data transmission $S_{i}=\{(j, i, k)\}$ are allowed at node $i$. In two-way network coding, the coding and decoding requirements are equivalent. It is the coded rate of a commodity should be smaller than the input rate on the two-hop link. Corresponding constraints are given as

$$
\begin{gathered}
\sum_{S_{i} \in F_{i}} \hat{g}_{S_{i}}^{c}-\sum_{(h, j) \in E} \sum_{\substack{S_{j} \in F_{j} \\
(h, j, i) \in S_{j}}} \hat{g}_{S_{j}}^{c}=T \sigma_{i}^{c}, \quad \forall i, c \\
\sum_{k \in V} \sum_{\substack{S_{i} \in F_{i}^{2} \\
(j, i, k) \in S_{i}}} \hat{g}_{S_{i}}^{c} \leq \sum_{h \in V} \sum_{\substack{S_{j} \in F_{j} \\
(h, j, i) \in S_{j}}} \hat{g}_{S_{j}}^{c} j \neq i, \forall i, c \\
\sum_{c \in C} \sum_{S_{i} \in F_{i}} e_{S_{i}}^{t} \hat{g}_{S_{i}}^{c} /\left|S_{i}\right|+\sum_{c \in C} \sum_{(h, j) \in E} \sum_{\substack{S_{j} \in F_{j} \\
(h, j, i) \in S_{j}}} e_{j i}^{r} \hat{g}_{S_{j}}^{c} \leq E_{i}, \quad \forall i
\end{gathered}
$$

where equation (11) is the flow conservation law. The constraint in equation (12) is the coding and decoding requirements of two-way network coding, which shows that the total transfer rate of commodity $c$ on the two-hop link $(j, i, k), \forall k$, should be less than the total input flow rates of commodity $c$ from $j$ to $i$. Energy constraint is given in (13).

\section{Overhearing Network Coding}

Besides the requirement of two-way network coding, overhearing from previous hop nodes is considered here. To correctly decode packets with overhearing, the overheard rate from the other previous hop nodes should be larger than the total coded rate.

$$
\sum_{S_{i} \in F_{i}} \hat{g}_{S_{i}}^{c}-\sum_{(h, j) \in E} \sum_{\substack{S_{j} \in F_{j} \\(h, j, i) \in S_{j}}} \hat{g}_{S_{j}}^{c}=T \sigma_{i}^{c}, \quad \forall i, c
$$




$$
\begin{gathered}
\sum_{k \in V} \sum_{S_{i},(j, i, k) \in S_{i}} \hat{g}_{S_{i}}^{c} \leq \sum_{h \in V} \sum_{\substack{S_{j} \in F_{j} \\
(h, j, i) \in S_{j}}} \hat{g}_{S_{j}}^{c} j \neq i, \forall i, c \\
\sum_{S_{i} \in F_{i}^{o}} \hat{g}_{S_{i}}^{c} \leq \sum_{h \in V} \sum_{S_{j}=\left\{\left(h, j^{\prime}, i\right)\right\}} \hat{g}_{S_{j}}^{c} \forall i, c,(j, i, k),\left(j^{\prime}, i, k^{\prime}\right) \in S_{i}, k \neq j^{\prime} \\
\sum_{c \in C}\left(\sum_{S_{i} \in F_{i}} \hat{g}_{S_{i}}^{c} e_{S_{i}}^{t}+\sum_{j \in V} \sum_{\substack{S_{j} \in F_{j} \\
i \in n\left(S_{j}\right)}} \hat{g}_{S_{j}}^{c} e_{j i}^{r}+\sum_{j \in V} \sum_{S_{j} \in F_{j}^{o}} \hat{g}_{S_{j}}^{c} e_{j i}^{r}\right) \leq E_{i}, \quad \forall i
\end{gathered}
$$

where equation (14) is the flow conservation law. Equation (15) is the coding requirement: the total coding rate of commodity $c$ including two-hop link $(j, i, k), \forall k$ is less than the total input rate from node $j$.

Equations (16) describes decoding requirements of network coding with overhearing. The sum rate of all coding scheme $S_{i}$ including $(j, i, k)$ and $\left(j^{\prime}, i, k^{\prime}\right), k \neq j^{\prime}$ is less than the uncoded data rate $\sum_{h \in N} \sum_{S_{j}=\left\{\left(h, j^{\prime}, i\right)\right\}} \hat{g}_{S_{j}}^{c}$ from $j^{\prime}$ to $i$ and overheard by $j$. Equation (17) is the energy constraint.

\section{Flow Augmenting Routing with Network Coding}

In this section, We first describe flow augmenting routing (FA) presented by Chang, et al [13], and then extend it to FA with network coding (FANC).

For each commodity $c$ with traffic demand $x^{c}$ in $C$, the lowest cost path $P_{c}$ from the source $s^{c}$ to the sink node $d^{c}$ is found first, and then $\lambda x^{c}$ flow is added to each link in path $P_{c}$, where $\lambda$ is the flow augmenting step size. Then the residual energy status of each node is updated, and the progress is continued until one node in the network is out of energy. Since the lowest cost path can be found with many distributed and efficient routing algorithms, such as the Bellman-Ford algorithm [14], flow augmenting routing is very extendable.

When network coding is considered, we do one-hop network coding according to different network coding schemes allowed after the flow is augmented, and the residual energy is calculated according to the cost with network coding.

The cost $z_{i j}$ of the link $(i, j)$ is defined as

$$
z_{i j}=\left(e_{i j}^{t}\right)^{x_{1}} \underline{E}_{i}^{-x_{2}} E_{i}^{x_{3}}+\left(e_{i j}^{r}\right)^{x_{1}} \underline{E}_{j}^{-x_{2}} E_{j}^{x_{3}}
$$

where $E_{i}, \underline{E}_{i}$ are the initial and the residual energy after flow augmenting of node $i . x_{1}, x_{2}$, and $x_{3}$ are weights of parameters $e_{i j}^{t}, \underline{E}_{i}$, and $E_{i}$, respectively.

The corresponding cost of a path $P$ is

$$
Z_{P}=\sum_{(i, j) \in P} z_{i j}
$$

The details of flow augmenting routing with network coding (FANC) is given in Algorithm 1.

\section{Performance Evaluation}

\section{A. Simulation Setup}

Similar to that in [13], we can assume the energy consumption per unit information transmission from node $i$ to $j$ as

$$
e_{i j}^{t}=e^{T}+\epsilon d_{i j}^{4}
$$

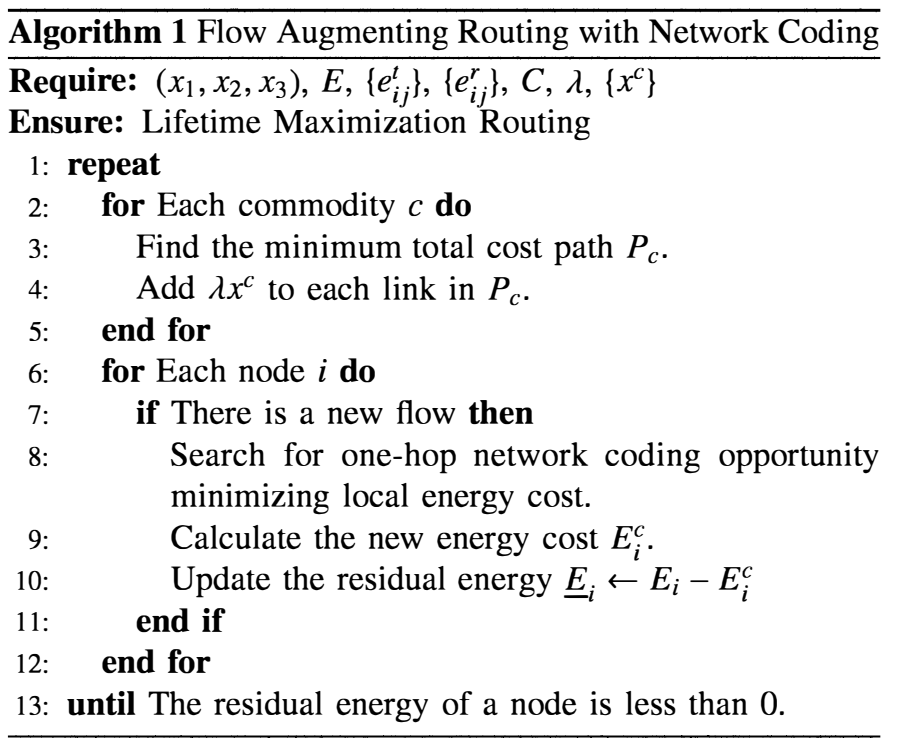

and

$$
e_{j i}^{r}=e^{R}
$$

where $e^{T}=50 \mathrm{~nJ} / \mathrm{bit}, e^{R}=150 \mathrm{~nJ} / \mathrm{bit}$ are the constant energy cost of the transceiver for transmission and receiving respectively, $\epsilon=0.1 \mathrm{~nJ} / \mathrm{bit} / \mathrm{m}^{4}$ is the energy cost of transmitting one bit information one meter [13]. In the physical power control model, $d_{i j}$ is the real distance between nodes $i$ and $j$, while in the protocol model, $d_{i j}$ is equal to $15 \mathrm{~m}$. According to results in [13], the step $\lambda$ used for both FA and FANC is selected as 5000 bits here.

Before performance simulation, we have evaluated the influence of $\left(x_{1}, x_{2}, x_{3}\right)$ with simulation in a random topology, and $\left(x_{1}, x_{2}, x_{3}\right)=(1,20,20)$ and $=(1,30,30)$ are selected for twoway and overhearing network coding respectively. For space limitation, details are omitted here.

\section{B. Random Topology}

In the following, we evaluate the lifetime performance of FANC according to the number of traffic demands in a random topology. Due to space limitation, we only give the results of one random topology as shown in Figure 2. We place all nodes in a $50 \mathrm{~m} \times 50 \mathrm{~m}$ area, randomly allocate initial energy between 10 and 20 joules, choose source and destination nodes randomly according to the number of flow, and set all traffic demands to 0.4 . The simulation is run for 30 times, and the normalized lifetimes under the protocol and physical models are shown in Figures 3 and 4, respectively.

From these figures, we can find the following observations. Under the protocol model, the normalized lifetime increases with the number of flow demands. Under the physical model, the normalized lifetime increases first, and then decreases. The performance gain with network coding under the protocol model is much higher than that under the physical model.

It is reasonable and explained as follows. Under the protocol model, the energy cost is proportional to the number of transmissions, and the number of possible network coding 


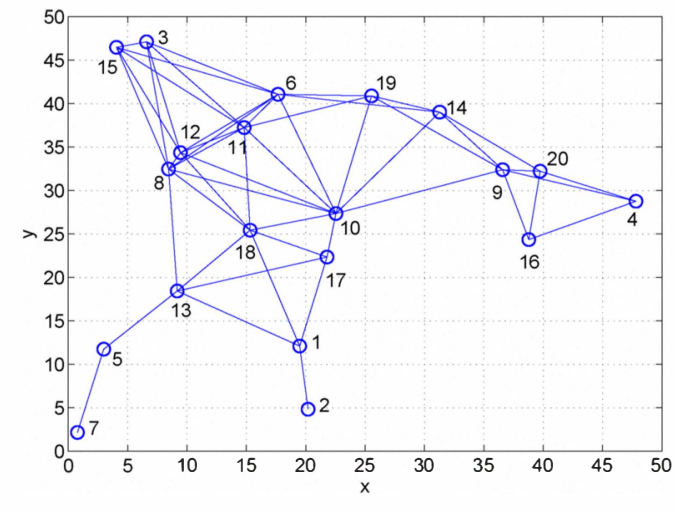

Fig. 2. A random topology with 20 nodes in a $50 \mathrm{~m} \times 50 \mathrm{~m}$ area.

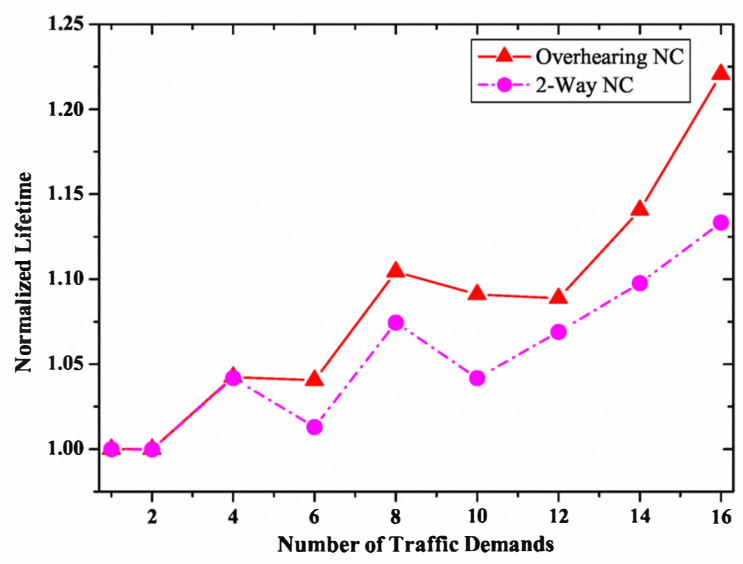

Fig. 3. Normalized lifetime under the protocol model.

opportunities generally increases with the number of transmissions. Thus, the lifetime gain with network coding also increases accordingly. Under the physical model, when the number of traffic demands is relatively small, the lifetime extension increases with traffic demands due to network coding at intermediate nodes. However, the energy cost is dominated by the link with higher power in physical model, thus the ratio between the saved energy with network coding and dominated larger power cost decreases, and the lifetime gain with network coding also decreases as the number of traffic demands is larger than a threshold. The threshold depends on the network structure, from which the influence is not considered in this paper.

Note that in real networks, depending on physical-layer technologies, the normalized lifetime is between those under the protocol model without power control and the physical model with perfect power control.

\section{Conclusion}

In this paper, we investigated the lifetime maximization routing with network coding in wireless multihop networks. The problem was formulated into three optimization problems with no network coding, two-way network coding, and overhearing network coding. Then the flow augmenting routing with network coding (FANC) was proposed, and its perfor-

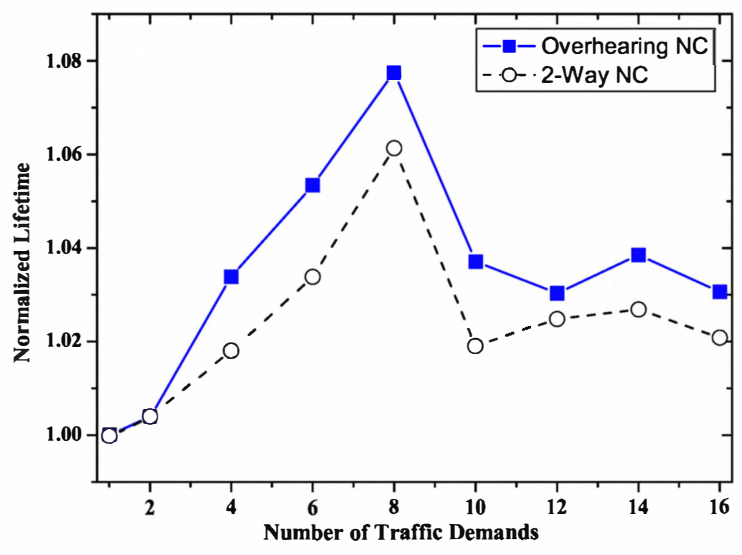

Fig. 4. Normalized lifetime under the physical model.

mance is evaluated in a randomly generated networks. The results show that, with network coding, the network lifetime can be extended up to $23 \%$ and $8 \%$ under the protocol and physical models, respectively. Furthermore, we also found that the lifetime improvement ratio increases as the number of traffic demands under the protocol model, while it increases first, and then decreases under the physical model.

\section{REFERENCES}

[1] J.-H. Chang, and L. Tassiulas, "Energy conserving routing in wireless ad-hoc networks," in Proc. IEEE INFOCOM, 2000.

[2] R. Madan, and S. Lall, "Distributed algorithms for maximum lifetime routing in wireless sensor networks," IEEE Trans. on Wireless Communications, vol. 5, no. 8, pp. 2185-2193, Aug. 2006.

[3] R. Ahlswede, N. Cai, S.-Y. R. Li, and R. W. Yeung, "Network information flow," IEEE Trans. on Information Theory, vol. 46, no. 4, pp. 1204-1216, Jul. 2000.

[4] S-Y. R. Li, r. W. Yeung, and N. Cai, "Linear network coding," IEEE Trans. on Information Theory, vol. 49, no. 2, pp. 371-381, Feb. 2003.

[5] T. Ho, M. Medard, and R. Koetter, "A random linear network coding approach to multicast," IEEE Trans. on Information Theory, vol. 52, no. 10, Oct. 2006.

[6] S. Katti, H. Rahul, W. Hu, D. Katabi, M. Medard, and J. Crowcroft, "XORs in the air: Practical wireless network coding," IEEE/ACM Trans. on Networking, vol. 16, no. 3, pp. 497-510, Jun. 2008.

[7] S. Sengupta, S. Rayanchu, and S. Banerjee, "An analysis of wireless network coding for unicast sessions: The case for coding-aware routing," in IEEE Proc. INFOCOM, May 2007.

[8] L. You, P. Wu, Z. Pan, H. Hu, M. Song, and J. Song, "Cross-layer optimization of wireless multi-hop networks with network coding," in Proc. International Conference for Wireless Communications and Signal Processing, 2009.

[9] Tao Cui, Lijun Chen and Tracey Ho, "Energy efficient opportunistic network coding for wireless networks," in INFOCOM, Phoenix, AZ, USA, Apr 2008, pp. 361-365.

[10] Nagajothy. M, and D. S. Radha, "Network lifetime enhancement in wireless sensor network using network coding," in International Conference on Control, Automation, Communication and Energy Conservation, Jun 2009.

[11] Y. Hong, J. Xu, and C. Jiang, "Lifetime maximization in wireless sensor networks with network coding," in Proc. IEEE WiCom, Sep 2007, pp. 2527-2530.

[12] A. Khreishah, C.-C. Wang, and N. B. Shroff, "Cross-layer optimization for wireless multihop networks with pairwise intersession network coding," JSAC, vol. 27, pp. 606-621, Jun. 2009.

[13] J.-Hoo, Chang, and L. Tassiulas, "Maximum lifetime routing in wireless sensor networks," IEEE/ACM Trans. Networking, vol. 12, no. 4, pp. 609619, Aug. 2004.

[14] D. Brown and R. Gallager, Data Networks, 2, Ed. New York: PrenticeHall, 1987. 